\title{
Strong Instability of Solitary Waves for Inhomogeneous Nonlinear Schrödinger Equations
}

\author{
chenglin Wang ${ }^{1}$ and Jian Zhang ${ }^{1}$ \\ ${ }^{1}$ University of Electronic Science and Technology of China
}

March 13, 2021

\begin{abstract}
This paper studies the inhomogeneous nonlinear Schrödinger equations, which may model the propagation of laser beams in nonlinear optics. Using the cross-constrained variational method, a sharp condition for global existence is derived. Then, by solving a variational problem, the strong instability of solitary waves of this equation is proved.
\end{abstract}

\section{Hosted file}

Strong instability of Solitary waves for Inhomogeneous NLS.pdf available at https: //authorea.com/users/401298/articles/513444-strong-instability-of-solitary-waves-forinhomogeneous-nonlinear-schr\%C3\%B6dinger-equations 